Cahiers
Recherche
surles Droits Fondamentaux
Cahiers de la recherche sur les droits fondamentaux

17 | 2019

La motivation des actes administratifs. Le droit français à la lumière du droit administratif comparé

\title{
La motivation des actes administratifs en droit allemand
}

Reine Wakote

\section{OpenEdition}

Journals

Édition électronique

URL : https://journals.openedition.org/crdf/3700

DOI : $10.4000 /$ crdf. 3700

ISSN : 2264-1246

Éditeur

Presses universitaires de Caen

Édition imprimée

Date de publication : 31 décembre 2019

Pagination : 57-68

ISBN : 978-2-84133-960-0

ISSN : $1634-8842$

Référence électronique

Reine Wakote, "La motivation des actes administratifs en droit allemand », Cahiers de la recherche sur les droits fondamentaux [En ligne], 17| 2019, mis en ligne le 06 février 2021, consulté le 15 novembre 2022. URL : http://journals.openedition.org/crdf/3700 ; DOI : https://doi.org/10.4000/crdf.3700 


\title{
La motivation des actes administratifs en droit allemand
}

\author{
Reine WAKOTE \\ Maîtresse de conférences en droit public à l'université de Lorraine \\ Institut de recherches sur l'évolution de la nation et de l'État (IRENEE, EA 7303)
}

I. Les conditions d'applicabilité de l'exigence de motivation
A. La diversité des actes administratifs concernés
B. Les caractéristiques tenant à l'acte administratif

II. La mise en œuvre de la motivation
A. Le contenu et la forme de la motivation
B. La variabilité du degré de motivation
C. Les exceptions à l'exigence de motivation

III. La portée de l'exigence de motivation
A. La distinction entre l'irrégularité et la nullité de l'acte administratif dépourvu de motivation
B. Le possible "rétablissement " de la motivation ou la "guérison " de l'acte administratif

Jusqu'à l'adoption de la loi du 25 mai 1976 relative à la procédure administrative (Verwaltungsverfahrensgesetz - VwVfG) ${ }^{1}$, les règles relatives à la procédure menée devant les autorités administratives faisaient l'objet de textes épars. Cette loi transpose ainsi le Code [fédéral] de procédure administrative en Allemagne. Mais la codification opérée par la loi de 1976 ne constitue qu'une codification partielle, tant de nombreux domaines d'action de l'autorité administrative font l'objet de textes spécifiques ${ }^{2}$. Les règles générales relatives à la motivation des actes administratifs sont aménagées au sein du $\$ 39$ VwVfG. Cette disposition est composée de deux subdivisions. La première, le $\$ 39$ I VwVfG, prévoit les conditions dans lesquelles une motivation doit être énoncée; tandis que la seconde, le $\$ 39$ II VwVfG, prévoit les situations pour lesquelles la motivation n'est pas requise.

Selon l'approche de la doctrine allemande, si la motivation assure trois fonctions fondamentales, curieusement,

1. Loi du 25 mai 1976, BGBl. I, 29 mai 1976, p. 1253. Cette loi est consultable en ligne dans sa version d'origine : https://www.bgbl.de/xaver/bgbl/start. xav?start=//*\%5B@attr_id=\%27bgbl176s1253.pdf\%27\%5D\#_bgbl_\%2F\%2F*\%5B\%40attr_id\%3D\%27bgbl176s1253.pdf\%27\%5D_1554973705583. Elle a subi, depuis, deux modifications importantes : celle opérée par la loi du 23 janvier 2003 (BGBl. I, 29 janvier 2003, p. 102) et la dernière par la loi du 18 décembre 2018 (BGBl. I, 21 décembre 2018, p. 2639). La version consolidée, modifiée par la loi de 2018, est consultable en ligne: https:// www.gesetze-im-internet.de/vwvfg/VwVfG.pdf. Pour un aperçu des travaux de la commission de codification, voir F.-O. Kopp, U. Ramsauer, Verwaltungsverfahrensgesetz: Kommentar, $19^{\mathrm{e}}$ éd., Munich, C. H. Beck, 2018, «Einführung I", ${ }^{\text {os }} 1-9$ et n ${ }^{\text {os }} 25-35$ g. Toutes les traductions de cette loi sont les nôtres. Voir, en fin d'article, la liste des sigles utilisés dans les références.

2. Voir notamment le Code de l'action sociale (Sozialgesetzbuch-SGB) institué par la loi du 23 décembre 1976 (BGBl. I, 30 décembre 1976, p. 3845 ); le Code de procédure fiscale (Abgebeordnung - AO), institué par la loi 16 mars 1976 (BGBl. I, 23 mars 1976, p. 613). 
elle n'est pas appréhendée comme ayant pour fonction première d'assurer la protection des droits des citoyens ${ }^{3}$.

Tout d'abord, la première fonction de la motivation consiste en la participation à la concrétisation du principe de l'État de droit en Allemagne. Cette conception n'est pas étonnante en soi, et demeure liée aux vicissitudes des exactions du régime national-socialiste durant la Seconde Guerre mondiale. Après 1945, les juristes allemands ayant participé à la reconstruction de l'État allemand sur le terrain du droit se sont efforcés de doter le principe de l'État de droit d'un contenu matériel ${ }^{4}$, à travers les droits fondamentaux, et d'asseoir le principe de démocratie en Allemagne. Par voie de conséquence, l'exigence de motivation contribue à assurer la protection procédurale non seulement des droits fondamentaux mais aussi des droits publics subjectifs. En effet, l'article $1^{\text {er }}$ I de la Loi fondamentale met en avant la dignité de la personne humaine que tous les pouvoirs publics doivent respecter et protéger ${ }^{5}$. Les administrés ne sont plus seulement considérés comme de simples sujets (Untertan) de l'action administrative mais, désormais, comme des citoyens (Bürger) auxquels des droits sont reconnus et dont la protection juridictionnelle est garantie par l'article $19 \mathrm{IV}$ de la Loi fondamentale. Ce faisant la motivation ne constitue ni un droit fondamental, ni un droit public subjectif, mais incarne un outil qui intervient pour faciliter l'accès aux voies de droit. Elle permet aux citoyens de connaître les éléments de fait et de droit retenus par toute autorité administrative pour adopter une décision les concernant et, ainsi, d'apprécier l'opportunité de saisir ou non le juge administratif ${ }^{6}$. Elle incarne ainsi un outil qui garantit le "principe d'égalité des armes en matière procédurale» (Grundsatz der Verfahrensfairness) ${ }^{7}$.

Ensuite, la deuxième fonction de la motivation de l'acte administratif consiste en un développement de la «bonne administration» (gute Verwaltung). Elle inscrit l'administration dans une perspective d'administration moderne qui, dans ses relations avec les citoyens, doit les considérer comme des «partenaires» et non plus comme des sujets. La motivation insère l'autorité administrative, antérieurement toute puissante, dans le principe de démocratie et constitue une exigence qui doit guider son action au regard de ce principe et lui permettre d'exercer un "autocontrôle» (Selbstkontrolle) tant de ses modalités d'action que de ses agents ${ }^{8}$. La motivation permet de s'assurer que les autorités administratives agissent, dans un État de droit, conformément à la légalité et que leurs actions sont conformes aux faits relevés et aux buts poursuivis. Il en résulte que la motivation joue aussi une "fonction d'acceptation" (Akzeptanzfunktion) par les citoyens de l'action menée par l'administration?.

Enfin, il résulte de ces considérations que l'exigence de motivation assure pour troisième fonction la sécurité juridique (Rechtssicherheit) ${ }^{10}$.

L'aménagement de l'exigence de motivation des actes administratifs intègre l'ensemble de ces considérations qu'il tente de concilier: il ne s'agit pas d'alourdir l'action de l'autorité administrative, mais, tout en lui offrant la latitude nécessaire, puisqu'elle intervient d'abord dans un but d'intérêt général, d'inscrire cette action dans le cadre du droit, d'une part, et d'offrir d'autre part aux administrés-citoyens le moyen de la protection de leurs droits, lorsque ce cadre n'est pas respecté.

Cet aménagement sera abordé sous trois aspects, en évoquant tout d'abord les conditions d'applicabilité de l'exigence de motivation (I), ensuite en cernant les modalités selon lesquelles celle-ci est mise en œuvre (II) et enfin en appréhendant sa portée (III).

\section{Les conditions d'applicabilité de l'exigence de motivation}

Le $\$ 39$ I phr. 1 VwVfG dispose qu' « un acte administratif écrit ou électronique ou confirmé par écrit ou par voie électronique doit être motivé ${ }^{11}$. Cette disposition énonce une diversité d'actes administratifs soumis à l'exigence de motivation (A) dont il conviendra de cerner les caractéristiques pour parvenir à une délimitation précise (B).

\section{A. La diversité des actes administratifs concernés}

Selon le $\$ 39$ I phr. 1 VwVfG, la motivation vise les actes administratifs écrits ou électroniques d'une part ou les

3. F.-O. Kopp, U. Ramsauer, Verwaltungsverfahrensgesetz: Kommentar, $\S 39, \mathrm{n}^{\text {os }} 4-6$.

4. En effet, le principe de l'État de droit, apparu à la fin du XIX ${ }^{\mathrm{e}}$ siècle, a principalement servi à asseoir le constitutionnalisme sur les terres allemandes, désormais unifiées par Bismarck, suite à la défaite de Sedan. Il a ainsi incarné l'outil à l'aune duquel la répartition des compétences entre la Fédération et les États fédérés devait être opérée.

5. Art. $1^{\text {er }} \mathrm{I}$ de la Loi fondamentale : «La dignité humaine est inviolable. Tous les pouvoirs sont tenus de la respecter et de la protéger» («Die Würde des Menschen ist unantastbar. Sie zu achten und zu schützen ist Verpflichtung aller staatlichen Gewalt»).

6. P. Becker, «Überlegungen zur 'Neuzeit des Prüfungsrechts'», NVwZ, 1993, p. 1130 et 1134. BVerwGE ( 28 novembre 1980, n $^{\circ}$ C 24.78$), 61,200$ (205); BVerwGE (9 décembre 1992, nº 6 C 3.92), 91, 262 (\$ 18 et 33), NVwZ, 1993, p. 679.

7. T. Müller-Ibold, Die Begründungspflicht im europäischen Gemeinschaftsrecht und im deutschen Recht: eine rechtsvergleichende Untersuchung, Francfort-sur-le-Main, P. Lang, 1990, p. 138

8. A. Wittern, M. Baßlsperger, Verwaltungs- und Verwaltungsprozessrecht: Grundriss für Ausbildung und Praxis, $20^{\mathrm{e}}$ éd., Stuttgart, Kohlhammer, 2016, $\$ 264$; U. Kischel, Folgen von Begründungsfehlern: Verwaltungsprozeß, Zivilprozeß, Verwaltungsverfahren, Tübingen, Mohr Siebeck, 2004, p. 8.

9. F.-O. Kopp, U. Ramsauer, Verwaltungsverfahrensgesetz: Kommentar, $\$ 39, \mathrm{n}^{\circ} 4$.

10. T. Müller-Ibold, Die Begründungspflicht..., p. 141; F.-O. Kopp, «Die Bestandskraft von Verwaltungsakten», DVBl, 1983, p. 392 (p. 397 ).

11. $\$ 39 \mathrm{I}$ phr. $1 \mathrm{VwVfG}$ : «Ein schriftlicher oder elektronischer sowie ein schriftlich oder elektronisch bestätigter Verwaltungsakt ist mit einer Begründung zu versehen». 
actes administratifs confirmés par écrit ou par voie électronique d'autre part. Au regard de l'aménagement du $\$ 39$ I phr. 1 VwVfG, le caractère électronique de l'acte n'est donc pas un obstacle à la motivation, dès lors que le $\$ 37$ II phr. 1 VwVfG prévoit qu’ « un acte administratif peut être adopté aussi bien par écrit, par voie électronique, oralement ou autrement $»^{12}$.

Les actes administratifs écrits constituent la règle et présentent des avantages en matière de preuve... tant pour l'autorité administrative que pour leurs destinataires. Toutefois, le législateur peut prévoir une forme particulière pour certains actes écrits, notamment en cas de nomination ou d'affectation d'un fonctionnaire ${ }^{13}$.

En ce qui concerne les actes administratifs électroniques, il ne s'agit pas spécifiquement d'actes rédigés par voie électronique, mais de ceux dont l'adoption peut être programmée: il ne suffit pas que l'acte ait été rédigé à l'aide d'un ordinateur, mais qu'il ait été adopté suite à une programmation électronique. Cela concerne essentiellement les actes administratifs résultant de formulaires ${ }^{14}$ établis par l'autorité administrative.

Qu'il s'agisse d'actes administratifs écrits ou électroniques, leur auteur doit être identifiable. En ce sens, l'autorité administrative compétente pour les édicter doit mentionner, dans les actes, les éléments permettant à leurs destinataires de s'en assurer, conformément au $\S 37$ III VwVfG ${ }^{15}$.

\section{B. Les caractéristiques tenant à l'acte administratif}

En premier lieu, la question se pose de savoir si la motivation ne s'applique qu'aux seuls actes pourvus d'un contenu matériel ou si elle s'impose aussi aux actes procéduraux. Le droit allemand n'opère pas cette distinction et ne s'attache pas à la question de savoir si l'acte modifie ou non le droit applicable à leur destinataire. La question de savoir si l'acte décide véritablement, et incarne donc, au regard de la terminologie usité en droit français, une décision stricto sensu, emportent peu d'effet, contrairement au droit français. Toutefois, les actes qui se rapportent strictement à une procédure menée par l'autorité administrative ne sont pas soumis à l'obligation de motivation. Sauf s'ils s'avèrent, dans leur résultat, emporter des effets sur la situation juridique de l'administré. Dans ce cas, la motivation est requise.

En deuxième lieu, en droit allemand, la motivation n'est pas conditionnée à la question de savoir si l'acte administratif en cause est susceptible de recours ou non. Cela résulte de l’interprétation du $\$ 44$ a phr. 1 de la loi sur la juridiction administrative (VerwaltungsgerichtsordnungVwGO), relatif aux actes de procédure menée par l'autorité administrative ${ }^{16}$. De même, n'entre pas en considération le fait de savoir si l'acte est favorable (begünstigender) ou défavorable (belastender) à son destinataire, contrairement au droit français ${ }^{17}$.

En troisième lieu, les décisions ou avis émis par des instances collégiales sont considérés comme des actes préparatoires (vorbereitende Entscheidungen) et non comme des actes administratifs; ils échappent de ce fait à l'obligation de motivation. Toutefois, les actes administratifs résultant de tels décisions ou avis collégiaux sont aussi soumis à l'obligation de motivation. Il appartient à l'autorité compétente pour adopter l'acte administratif définitif de procéder à cette motivation, sauf si un texte en dispose autrement ${ }^{18}$. Si l'autorité compétente demeure liée au sens de l'avis ou de la décision collégiale, elle doit motiver en intégrant les divergences ou les pluralités de point de vue ainsi émises. Parfois, les textes imposent à l'autorité compétente de motiver en reprenant la motivation de l'instance collégiale, si cette dernière était tenue de motiver sa décision ou son avis ${ }^{19}$. Enfin, dans certaines circonstances, cette motivation sera requise eu égard aux intérêts en présence. Tel est le cas pour les comités élisant les juges ${ }^{20}$.

12. $\$ 37 \mathrm{II}$ phr. 1 VwVfG: «Ein Verwaltungsakt kann schriftlich, elektronisch, mündlich oder in anderer Weise erlassen werden».

13. Voir ainsi le $\$ 8$ II du Code de la fonction publique (Beamtenstatusgesetz - BeamtStG); H. Maurer, Verwaltungsrecht, $19^{\mathrm{e}}$ éd., Munich, C. H. Beck, $2018, \$ 10, n^{\circ} 41$

14. T. Müller-Ibold, Die Begründungspflicht..., p. 180 .

15. Selon le $\$ 37$ III VwVfG: «L’acte administratif écrit ou électronique doit identifier l'autorité émettrice et contenir la signature ou le nom du responsable de l'autorité, de son supérieur hiérarchique ou de son représentant. Lorsqu'un texte prévoit qu'un acte administratif peut être adopté sous la forme électronique, le certificat permettant d’identifier l'autorité administrative compétente doit être inséré. Dans le cadre du $\$ 3$ a II phr. $4 \mathrm{n}^{\circ} 3$ [en cas de confirmation d'un échange électronique], la confirmation des courriels reçus doit permettre de reconnaître l'adresse mail de l'autorité émettrice, conformément au $\$ 5$ de la loi relative au courrier électronique (De-Mail-Gesetz)» («Ein schriftlicher oder elektronischer Verwaltungsakt muss die erlassende Behörde erkennen lassen und die Unterschrift oder die Namenswiedergabe des Behördenleiters, seines Vertreters oder seines Beauftragten enthalten. Wird für einen Verwaltungsakt, für den durch Rechtsvorschrift die Schriftform angeordnet ist, die elektronische Form verwendet, muss auch das der Signatur zugrunde liegende qualifizierte Zertifikat oder ein zugehöriges qualifiziertes Attributzertifikat die erlassende Behörde erkennen lassen. Im Fall des $\S_{3} a$ Absatz 2 Satz 4 Nummer 3 muss die Bestätigung nach $\$ 5$ Absatz 5 des De-Mail-Gesetzes die erlassende Behörde als Nutzer des De-Mail-Kontos erkennen lassen»).

16. BVerwG (22 septembre 2016, no 2 C 16.15), NVwZ, 2017, p. 489; DÖV, 2017, p. 212; note M. Stuttmann, RÜ2, 2017, p. 47. Voir H.-J. Knack, H.-G. Henneke, Verwaltungsverfahrensgesetz: Kommentar, $10^{e}$ éd., Cologne, C. Heymann, 2014, $\$ 39, n^{\circ} 10$.

17. En effet, en France, la loi du 11 juillet 1979 relative à la motivation des actes administratifs et à l'amélioration des relations entre l'administration et le public disposait que «les personnes physiques ou morales ont le droit d'être informées sans délai des motifs des décisions administratives individuelles défavorables qui les concernent». Bien que cette loi ait été abrogée, la codification opérée, en 2016, dans le Code des relations entre le public et l'administration (CRPA) maintient l'exigence de la motivation au caractère défavorable de la décision.

18. R. Dolzer, «Zum Begründungsgebot im geltenden Verwaltungsrecht», DÖV, 1985, p. 17; H.-J. Knack, H.-G. Henneke, Verwaltungsverfahrensgesetz: Kommentar, $\$ 39, \mathrm{n}^{\circ} 16$.

19. T. Müller-Ibold, Die Begründungspflicht..., p. 223; R. Dolzer, «Zum Begründungsgebot... », p. 17

20. BVerwGE (19 juin 1997) 105, 89 (92) (Richterwahlausschuss). 
En quatrième lieu, le $₫ 39$ VwVfG n'exige pas de motivation pour les actes verbaux, sauf si l'intéressé en sollicite la confirmation et par là même la motivation, en excipant des dispositions du $\$ 37 \mathrm{II}$ phr. $2 \mathrm{VwVfG}^{21}$. Les actes provenant de signes ou de gestuelles émis par une autorité administrative, notamment par un agent régulant la circulation, se voient appliquer un régime identique. La confirmation de tels actes peut être opérée aussi bien par écrit que par voie électronique ${ }^{22}$.

En dernier lieu, les promesses énoncées par l'autorité administrative et aménagées au $\$ 38 \mathrm{VwVfG}$ sont aussi soumises à l'exigence de motivation ${ }^{23}$.

Au regard des conditions d'applicabilité posées pour la motivation, le droit allemand semble adopter une conception bien plus souple que le droit français, s'agissant des actes administratifs soumis à une telle obligation. Cette souplesse doit s'apprécier par rapport aux conditions mêmes de mise en œuvre de la motivation.

\section{La mise en œuvre de la motivation}

Le $\$ 39$ VwVfG aménage les modalités selon lesquelles l'autorité administrative doit procéder à la motivation. Ces modalités portent sur la forme et le contenu de la motivation et incarnent le cadre général de la motivation (A). Toutefois, la disposition prévoit un cadre variable pour la motivation des actes administratifs, laquelle demeure susceptible de degrés eu égard à la nature de la compétence administrative (B). Et enfin, elle énonce les cas pour lesquels la motivation n'est pas exigée (C).

\section{A. Le contenu et la forme de la motivation}

Le $\$ 39$ I phr. 2 VwVfG aménage les modalités selon lesquelles l'autorité administrative doit procéder à la motivation en ces termes: «La motivation comporte les éléments essentiels de fait et de droit sur lesquels l'autorité administrative s'est fondée ${ }^{24}$.

La doctrine considère que cette disposition impose d'apprécier la motivation tant au regard de la forme que du contenu. Mais, en réalité, le $\$ 39$ I phr. 2 VwVfG impose seulement une obligation de motivation formelle, en ce sens que la motivation doit contenir l'énonciation des éléments de fait et de droit à la base de la décision adoptée par l'autorité administrative. La motivation est censée établir l'appréciation des faits opérée par elle pour parvenir à la solution adoptée dans l'acte administratif. Ceci afin de permettre à l'intéressé de sonder les éventuelles voies de droit qui lui sont ouvertes ${ }^{25}$.

En premier lieu, s'agissant du contenu de la motivation, celui-ci ne s'entend pas au sens matériel. Tout d'abord, le contenu de la motivation signifie que la motivation doit traduire une cohérence entre les motifs de faits et de droit retenus à la base de la décision. Il ne doit pas y avoir de contradiction entre les motifs retenus, de sorte que la motivation soit intelligible (nachvollziehbar) pour le destinataire de l'acte. Le caractère intelligible de la motivation implique d'une part que le destinataire de l'acte soit en mesure d'en saisir "aisément " (ohne weiteres ${ }^{26}$ le sens, sans avoir à se référer à l'ensemble des échanges et correspondances intervenus durant la procédure; à défaut la motivation est irrégulière ${ }^{27}$. Ensuite, cela signifie que l'autorité administrative ne doit pas s'arrêter devant les difficultés d'interprétation, mais, au contraire, intégrer l'ensemble des éléments se rapportant à l'espèce. L'interprétation doit être conforme à la Loi fondamentale et aux éventuels droits fondamentaux en présence (voir II.B). Il ne s'agit pas, au demeurant, pour l'autorité administrative de faire état de la réflexion qu'elle a menée, des hésitations ou des certitudes qu'elle aurait pu avoir ou non face à tel ou tel motif. Mais il s'agit pour elle d'énoncer les raisons et les motifs qui l'ont convaincue d'adopter la décision retenue, ceux sur lesquels elle a fondé son opinion ${ }^{28}$. Par conséquent, l'interprétation qu'elle a privilégiée doit être exposée dans la motivation. Enfin, sur le terrain du contenu de la motivation, la jurisprudence de la Cour administrative fédérale affirme que, si l'autorité administrative a évacué certaines allégations ou points de vue des parties ou du destinataire de l'acte, elle doit en indiquer la cause dans la motivation, alors même que cela aurait pu aboutir à une autre solution ${ }^{29}$. La motivation ne peut donc pas être assise sur des éléments abstraits, mais s'attacher aux éléments concrets du cas d'espèce. À défaut, l'exigence de motivation n'est pas remplie $^{30}$. Mais paradoxalement, la jurisprudence

21. La doctrine procède ici à une lecture extensive et considère que la motivation s'applique aux actes verbaux, mais ne se concrétise qu'au moment de la confirmation sollicitée par l'intéressé. La jurisprudence adopte une lecture plus restrictive: VGH Mannheim, $N V w Z, 1989$, p. 164. La Cour administrative fédérale estime que c'est l'acte de confirmation de l'acte verbal qui impose la motivation et non l'acte verbal en lui-même: BVerwGE (19 juin 1997) (cité note précédente).

22. F.-O. Kopp, U. Ramsauer, Verwaltungsverfahrensgesetz: Kommentar, $\S 39, \mathrm{n}^{\circ} 8$.

23. Ibid., «Einführung I», $\mathrm{n}^{\text {os }} 1-9$, en particulier $\mathrm{n}^{\text {os }} 8-9$.

24. \$39 I phr. 2 VwVfG: «In der Begründung sind die wesentlichen tatsächlichen und rechtlichen Gründe mitzuteilen, die die Behörde zu ihrer Entscheidung bewogen haben».

25. OVG Bautzen (Saxe), 18 août 2006 (n 3 BS 138/o6), NVwZ, 2007, p. 847 et note M. Scheffer, « Begründungspflicht bei Personalentscheidungen », $N V w Z, 2007$, p. 779-782.

26. BVerwGE (24 novembre 1987), 83, 345 (357); J. Ziekow, Verwaltungsverfahrensgesetz: Kommentar, $3^{\mathrm{e}}$ éd., Stuttgart, Kohlhammer, 2013, $\$ 39$, ${ }^{\circ}$ 5.

27. BVerwG (14 avril 1992), NVwZ, 1992, p. 683.

28. U. Kischel, Die Begründung: zur Erläuterung staatlicher Entscheidungen gegenüber dem Bürger, Tübingen, Mohr Siebeck, 2003; U. Kischel, Folgen von Begründungsfehlern..., p. 8.

29. BVerwG, $D V B l, 1983$, p. 198 .

30. K. Obermayer, Grundzüge des Verwaltungsrechts und Verwaltungsprozeßrechts, $3^{\mathrm{e}}$ éd., Stuttgart, Boorberg, 1988 , p. 23. 
admet qu'une motivation puisse être standardisée, dès lors que la standardisation et les termes clés employés font suffisamment référence au cas concret ${ }^{31}$.

En deuxième lieu, la motivation ne repose pas sur les motifs de fait et de droit déterminants, mais sur ceux qui, restrictivement considérés, ont été déterminants ${ }^{32}$ pour l'adoption de l'acte administratif en cause, pour le cas d'espèce, même lorsque, dans leur résultat, ces motifs pourraient ne pas être soutenables (tragfähig) ${ }^{33}$. L'appréciation du caractère déterminant implique de prendre en considération les indices laissant supposer que les éléments de fait ou de droit n'auraient pas été suffisamment précisés, en amont, par le destinataire de l'acte, dans ses affirmations antérieures ${ }^{34}$. Si cette approche intègre l'idée même que le destinataire de l'acte doive être en mesure d'apprécier s'il peut ou non accepter l'acte administratif édicté ${ }^{35}$, étonnamment, le $\$ 39 \mathrm{VwVfG}$ n’exige pas expressément la motivation sur le plan matériel, sauf en cas de marge d'appréciation ou de pouvoir discrétionnaire reconnu à l'autorité administrative (voir II.B). Mais la conception de la motivation privilégiée, en droit allemand, manque ici de cohérence: car si les motifs retenus par l'autorité administrative doivent être soutenables, cela implique de s'assurer de la régularité de la motivation dans son aspect matériel; ce que le $\$ 39 \mathrm{VwVfG}$ n’exige pas. Au surplus, si les motifs ne sont pas soutenables, il en résulte, en droit allemand, une irrégularité matérielle de l'acte administratif et non une violation de l'obligation de motivation. La question se pose alors de savoir pourquoi l'autorité administrative doit être attentive au caractère soutenable de la motivation, puisque cela ne traduit pas un vice intrinsèque de la motivation énoncée.

En troisième lieu, la question subsiste de savoir si les éléments de procédure, notamment l'audition ou non de certaines personnes, le rejet de certaines expertises doivent figurer au sein de la motivation. La doctrine demeure partagée sur ce point. Si certains auteurs considèrent, «en principe ${ }^{36}$, que les questions liées à la procédure n'ont pas trait à la motivation telle que l'envisage le $\$ 39 \mathrm{VwVfG}$, d'aucuns sont d'avis que la motivation énoncée par l'autorité administrative doit intégrer les questions de procédure, lorsqu'elles ont été soulevées par les parties et fondent l'approche qu'ont celles-ci de l'issue du litige, ou encore lorsque la procédure menée par l'autorité administrative a pu influencer la solution finalement retenue ${ }^{37}$.
En dernier lieu, s'agissant du support de la motivation, il doit s'agir d'un acte administratif écrit ou d'un acte administratif électronique. Toutefois, la motivation ne saurait être énoncée sur une disquette ou sur une microfiche, ces supports n'étant pas considérés comme des actes administratifs. Au surplus, la motivation ne peut être réalisée par référence à des éléments contenus sur ces supports, ni par référence ou renvoi à des conversations téléphoniques auxquelles l'administration renverrait pour s'épargner toute motivation ${ }^{38}$.

Dans sa mise en œuvre, l'exigence de motivation demeure susceptible de variations, de degrés.

\section{B. La variabilité du degré de motivation}

Selon le $\$ 39$ I phr. 3 VwVfG:

La motivation de décisions prises sur le fondement d'un pouvoir discrétionnaire doit aussi contenir les arguments à partir desquels l'autorité administrative a fait usage de son pouvoir discrétionnaire ${ }^{39}$.

Cette disposition impose la motivation de l'acte administratif, lorsque l'autorité administrative s'est vue reconnaître un pouvoir discrétionnaire. La motivation demeure exigée eu égard à la nature de la compétence reconnue par les dispositions textuelles à la personne publique. En réalité, au regard de la jurisprudence administrative et constitutionnelle, la motivation est susceptible de variations : l'exigence de motivation oscille entre différents degrés, selon que la disposition octroie un pouvoir discrétionnaire à l'autorité administrative ou lui reconnaît une compétence liée. De prime abord cette exigence apparaît quelque peu atténuée en cas de pouvoir discrétionnaire et de compétence liée. Mais les solutions jurisprudentielles sont plus subtiles. Par ailleurs, la Cour administrative fédérale a énoncé des solutions éparses accentuant, en revanche, le degré d'exigence de motivation.

En premier lieu, s'agissant de la conception du pouvoir discrétionnaire, la langue allemande se déploie en finesse ${ }^{40}$. Le pouvoir discrétionnaire peut être expressément reconnu, notamment, lorsque le texte d'habilitation énonce plusieurs choix (pouvoir discrétionnaire de choix, Auswahlermessen), tous légaux, entre lesquels l'autorité administrative peut décider. Mais, parfois, les termes employés ne permettent pas de retenir qu'un pouvoir

31. BVerwGE (12 décembre 1973), 44, 215 (218).

32. BVerwGE (15 mai 1986), 74, 196 (205) et BVerwGE (9 décembre 1992), 91, 262 (268).

33. U. Kischel, Folgen von Begründungsfehlern..., p. 2.

34. F. Hufen, T. Siegel, Fehler im Verwaltungsverfahren, $5^{\mathrm{e}}$ éd., Baden-Baden, Nomos, 2013, p. 483.

35. U. Kischel, Folgen von Begründungsfehlern..., p. 9.

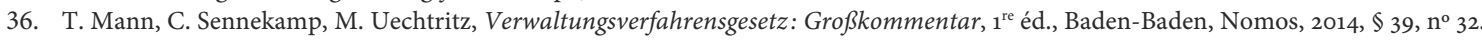

37. P. Stelkens, H. J. Bonk, M. Sachs et al., Verwaltungsverfahrensgesetz: Kommentar, $9^{\mathrm{e}}$ éd., Munich, C. H. Beck, 2018, $\$ 39$, n 53 ; F.-O. Kopp, U. Ramsauer, Verwaltungsverfahrensgesetz: Kommentar, $₫ 39, \mathrm{n}^{\circ} 18 \mathrm{a}$.

38. F.-O. Kopp, U. Ramsauer, Verwaltungsverfahrensgesetz: Kommentar, $\$ 39, \mathrm{n}^{\circ} 17$.

39. \$39 I phr. 3 VwVfG: «Die Begründung von Ermessensentscheidungen soll auch die Gesichtspunkte erkennen lassen, von denen die Behörde bei der Ausübung ihres Ermessens ausgegangen ist».

40. Voir, notamment, R. Wakote, Le contrôle du pouvoir discrétionnaire en France et en Allemagne depuis la fin du XIX ${ }^{e}$ siècle, thèse de doctorat en droit, sous la direction de M. Fromont, université Paris 1 - Panthéon-Sorbonne, 2011; H. Maurer, Verwaltungsrecht, $\$ 7$. 
discrétionnaire préexiste explicitement. Tel est le cas lorsque le texte applicable emploie le verbe "pouvoir» (können-Kannvorshrift) et s'analyse en une disposition potestative.

Tout d'abord, la motivation demeure, en principe, maintenue, lorsqu'une disposition octroie un pouvoir discrétionnaire. Cela se conçoit aisément, dans la mesure où le pouvoir discrétionnaire ainsi octroyé traduit une plus grande liberté de décision pour l'autorité administrative. La motivation permet alors de s'assurer qu'elle a fait un usage de son pouvoir discrétionnaire conforme (pflichtgemäß) à la loi d'habilitation et qu'elle n'a pas décidé de manière arbitraire (willkürlich).

Mais, si l'exigence de motivation persiste, même dans ces cas où l'autorité administrative dispose d'un pouvoir discrétionnaire ou d'une latitude pour décider, elle demeure atténuée. Dans ce cas, il importe que la motivation permette de relever que l'ensemble des intérêts en présence ont été intégrés dans l'appréciation à laquelle a procédé l'autorité administrative pour adopter sa décision ${ }^{41}$. L'administration doit aussi faire mention des éléments de faits sur lesquels elle s'est appuyée pour se prononcer, de sorte que le justiciable puisse d'une part s'assurer de ce qu'elle disposait, pour le cas d'espèce, d'un pouvoir discrétionnaire, et, d'autre part, du sens dans lequel elle l'a mis en œuvre. En définitive, la motivation doit traduire un usage du pouvoir discrétionnaire conforme au but pour lequel il a été octroyé ${ }^{42}$.

Ensuite, en cas de pouvoir discrétionnaire de choix (Auswahlermessen), l'autorité administrative est seulement tenue d'énoncer, dans la motivation, les différents intérêts déterminants en présence qu'elle a intégrés pour adopter sa décision ${ }^{43}$. Seule la nécessité absolue (Eilbedürftigkeit) peut la dispenser de motiver. Lorsque le choix porte sur la nomination de candidats à compétence équivalente ou comparable, l'autorité administrative dispose non seulement d'une marge d'appréciation pour évaluer les qualités requises des candidats, mais aussi d'un pouvoir discrétionnaire se limitant au choix final du candidat ${ }^{44}$.

Par ailleurs, la jurisprudence assimile aussi la «disposition-recommandation» (sollen, Sollvorschrift) ${ }^{45}$ à un pouvoir discrétionnaire et maintient l'exigence de motivation. La Cour administrative estime alors que la motivation de la décision doit, pour le cas d'espèce, contenir une appréciation des considérations déterminantes ainsi que les motifs qui la fondent ${ }^{46}$. L'autorité administrative doit, pour l'essentiel, s'attacher à ce que la motivation soit substanciée (substantiiert), cohérente (schlüssig) et intelligible (nachvollziehbar) ${ }^{47}$. Mais l'exigence de motivation est atténuée, puisqu'il ne s'agit pas d'énoncer les particularités des motifs déterminants, mais d'en exposer les grands traits ${ }^{4}$. D'autre part, lorsque le texte d'habilitation emploie le verbe "pouvoir en tant que disposition potestative» (können, Kannvorschrift), l'autorité administrative se voit reconnaître, en principe, toute latitude aussi bien pour décider du moment de son action, de ses modalités que de la décision elle-même ${ }^{49}$. La motivation est exigée dans les mêmes conditions que celles applicables en cas de disposition-recommandation ${ }^{50}$. Mais la disposition potestative peut évoluer et modifier la nature de la compétence de l'autorité administrative: la latitude pour décider se transforme en compétence liée dont les solutions sont exposées ci-dessous.

Ces solutions baroques semblent conforter une atténuation de l'exigence de motivation.

En revanche, lorsque subsiste un droit fondamental et que la personne publique doit décider sur la base d'un pouvoir discrétionnaire, ou de l'une des formes précédemment exposées, l'exigence de motivation se trouve renforcée. En cas de pouvoir discrétionnaire, la Cour administrative fédérale parle alors de "pouvoir discrétionnaire orienté» (intendiertes Ermessen) ${ }^{51}$. En cas de disposition potestative, celle-ci peut s'avérer, au final, ne laisser aucune latitude à l'autorité administrative, dès lors que, dans son résultat, son usage déboucherait sur la violation d'un droit fondamental. En définitive, en présence d'un droit fondamental, l'appréciation des éléments de fait et de droit de l'espèce aboutit, certes, à une solution donnée, mais, le droit fondamental l'emportant, une autre décision s'impose finalement ${ }^{52}$.

En second lieu, la motivation demeure aussi exigée en cas de compétence liée ${ }^{53}$.

41. BVerwGE (26 octobre 1978), 57, 1

42. T. Müller-Ibold, Die Begründungspflicht..., p. 312.

43. A. Wittern, M. Baßlsperger, Verwaltungs- und Verwaltungsprozessrecht..., $\$ 265$.

44. VGH Kassel, $N V w Z-R R, 1993$, p. 361.

45. BVerwGE (2o février 1990), 84, 375 (388); U. Kischel, Die Begründung..., p. 227 sq.

46. Ibid.

47. BVerwGE (9 mars 1990), 85, 36; BVerwGE (9 décembre 1992), 91, 262.

48. BVerwGE (9 décembre 1992), 91, 262 (265).

49. H. Maurer, Verwaltungsrecht, $\S 7, \mathrm{n}^{\text {os }}$ 9-12 et $\mathrm{n}^{\mathrm{os}} 49-50$.

50. Ibid.

51. BVerwGE (5 juillet 1985), 72, 1 (6) et BVerwGE (16 juin 1997), 105, 55 ( 57 sq.); U. Volkmann, «Das 'intendierte' Verwaltungsermessen », DÖV, 1996, p. 281; W. Hoppe, "Durch Soll-Vorschrift 'intendierte' Ziele der Raumordnung und ihre Bezüge zu 'intendiertem Ermessen'», in Staat, Kirche, Verwaltung. Festschrift für Hartmut Maurer zum 7o, Munich, C. H. Beck, 2001, p. 625; U. Kischel, Die Begründung..., p. 226-229.

52. Voir les références citées dans la note précédente.

53. Il est à signaler qu'une doctrine minoritaire considère, "en principe», que la compétence de l'autorité administrative est aussi liée, alors même que le texte applicable contient le verbe «devoir» (sollen - Sollvorschrift) dans le sens d'un conseil ou d'une recommandation. Ce n'est qu'exceptionnellement qu'elle peut disposer d'une latitude pour décider; voir T. Mann, C. Sennekamp, M. Uechtritz, Verwaltungsverfahrensgesetz: Großkommentar, $₫ 39, \mathrm{n}^{\circ} 32$; voir aussi F.-O. Kopp, U. Ramsauer, Verwaltungsverfahrensgesetz: Kommentar, $\S 28, \mathrm{n}^{\circ} 24$ et $\S 39, \mathrm{n}^{\circ} 38$. 
Tout d'abord, cela est le cas, notamment, avec l'emploi du verbe «devoir» (müssen, Mussvorschrift) dans le sens d'une obligation. Dès lors que la compétence de l'autorité administrative est liée, la motivation de l'acte administratif devra, en particulier, s'attacher à établir qu'elle a suivi une procédure régulière lors de l'adoption de l'acte. Cette lecture est cependant critiquée par une partie de la doctrine qui estime que le $\$ 39 \mathrm{VwVfG}$ aménage la motivation dans la première partie du Code de procédure administrative, laquelle ne traite que de l'acte administratif ayant un contenu matériel ${ }^{54}$ et non des actes procéduraux.

Ensuite, la motivation s'applique dans les mêmes conditions, lorsque la disposition potestative (können, Kannvorschrift) est liée à certaines exigences. Il en est ainsi, lorsque la disposition potestative prévoit que l'autorité administrative peut, entre plusieurs décisions de 1 à 10, adopter la décision 1, ou 2, ou 3, ou encore 4, si les conditions $\mathrm{A}, \mathrm{B}$ et $\mathrm{E}$ sont remplies. La réalisation desdites conditions lui octroie effectivement une latitude pour décider. Mais, a contrario, lorsque lesdites conditions ne sont pas remplies, l'autorité administrative voit sa compétence liée: elle est tenue d'adopter l'une des décisions de 5 à 10. Les exigences relatives à la motivation sont alors celles applicables en cas de compétence liée: la «disposition potestative» (können, Kannvorschrift) devient une «disposition obligatoire» (müssen, Mussvorschrift) ${ }^{55}$.

Enfin, les solutions retenues en cas de «décision orientée » (intendierte Entscheidung) révèlent un caractère singulier. La décision orientée traduit la situation dans laquelle les dispositions textuelles aménagent une règle générale pour laquelle, en définitive, une seule décision est possible ${ }^{56}$. Étonnamment, la jurisprudence assimile la décision orientée à la disposition-recommandation (sollen, Sollvorschrift) et elle lui transpose, en matière de motivation, les solutions de la disposition-recommandation précédemment exposées ${ }^{57}$. Mais, cette assimilation de la décision orientée à la disposition-recommandation apparaît quelque peu étrange, puisqu'une seule décision s'impose, finalement, et non un pouvoir discrétionnaire qui en découlerait comme en cas de disposition-recommandation. Il semble pourtant que la décision orientée présente plus de proximité avec la compétence liée: en effet le cadre de la décision est tellement orienté par les textes que l'autorité administrative est tenue d'adopter une seule décision; la décision se déploierait plutôt en un «devoir-obligation » (müssen, Mussvorschrift) ${ }^{58}$. D'autant plus qu'en cas de décision orientée, la motivation doit alors expressément s'appuyer sur les dispositions textuelles aménagées; le chemin de la motivation est préalablement balisé. Toutefois, l'autorité administrative peut s'en écarter de manière exceptionnelle; elle doit alors procéder à une motivation détaillée ${ }^{59}$, correspondant ainsi à une accentuation de l'exigence de motivation.

En troisième lieu, des solutions éparses de motivation peuvent être dégagées. En effet, la jurisprudence de la Cour administrative fédérale ${ }^{60}$ énonce, de manière constante, que le degré de motivation exigé varie aussi en fonction des particularités de l'espèce, des questions soulevées, de la nature des droits concernés, en particulier si des droits fondamentaux préexistent et, enfin, de la portée de la décision pour le justiciable. Une autre situation d'atténuation du degré de motivation concerne celle dans laquelle les textes applicables à l'espèce se concentrent sur le but à atteindre, énoncé comme élément déterminant ${ }^{61}$ de la décision à adopter. Ce but oriente la motivation à laquelle devra procéder l'autorité administrative: la motivation devra alors essentiellement traduire l'appréciation à laquelle elle a procédé au regard de ce but ${ }^{62}$. Il en résulte que l'exigence de motivation peut être soit accentuée, notamment lorsque la décision revêt un caractère d'exception ${ }^{63}$. Mais elle peut aussi être atténuée, lorsque les textes applicables au cas d'espèce se concentrent sur le but à atteindre et le désigne

54. T. Müller-Ibold, Die Begründungspflicht..., p. 190.

55. Pour une application jurisprudentielle, voir BVerwGE (8 décembre 1965), 23, 25 (29) et BVerwGE (7 février 1974), 44, 339 (342): emploi d'un «Kannvorschrift» duquel il découle pourtant une négation du pouvoir discrétionnaire de l'autorité administrative, aboutissant à lier sa compétence. Il convient aussi de souligner que la situation inverse peut être relevée. Ainsi en cas de disposition obligatoire (müssen, Mussvorschrift) liée à des conditions, prévoyant que l'autorité administrative doit, entre plusieurs décisions de 1 à 10, adopter la décision 1, ou 2, ou 3, ou encore 4, si les conditions $\mathrm{A}, \mathrm{B}$ et $\mathrm{E}$ sont remplies. La réalisation desdites conditions l'oblige effectivement à adopter l'une des décisions de 1 à 4 . A contrario, lorsque lesdites conditions ne sont pas remplies, l'autorité administrative retrouve une latitude pour adopter l'une des décisions de 5 à 10. Les exigences relatives à la motivation sont alors celles applicables en cas de pouvoir discrétionnaire: la disposition obligatoire (müssen, Mussvorschrift) est alors assimilable à une disposition potestative (können, Kannvorschrift) conférant une latitude pour décider ou encore à une dispositionrecommandation (sollen, Sollvorschrift); BVerwGE (17 mars 1992), 90, 88 (93): évolution d'une disposition obligatoire (müssen, Mussvorschrift) en disposition-recommandation (sollen, Sollvorschrift) et inversement.

56. BVerwGE (5 juillet 1985), 72, 1 (5).

57. BVerwGE (20 février 1990), 84, 375 et BVerwGE (9 décembre 1992), 91, 262 (265).

58. En réalité l'assimilation à laquelle procède la Cour administrative ne peut être admise qu'en matière de planification, où, parfois, la dispositionrecommandation (Sollvorschrift) peut évoluer en une disposition obligatoire (Mussvorschrift). En effet, dans ce cas, les recommandations émises sont tellement étoffées et contraignantes qu'il ne reste plus aucune latitude de décision au profit de l'autorité administrative; le pouvoir discrétionnaire qui devait résulter de la disposition-recommandation disparait et la compétence de l'autorité compétente s'articule, en pratique, en une compétence liée; BVerwGE (29 avril 1964), 18, 247. Mais, il convient de rappeler que la planification urbanistique se voit appliquer un régime spécifique de motivation aménagé au $\$ 74 \mathrm{I}$ phr. 2 VwVfG.

59. BVerwGE (25 septembre 1992), 91, 82 (90).

60. BVerwGE (12 octobre 1965), 22, 212 (217); BVerwGE (20 février 1990), 84, 375 (388 sq.); BVerwGE (9 décembre 1992), 91, 262 (268). Voir aussi F. Hufen, T. Siegel, Fehler im Verwaltungsverfahren, p. 472; F.-O. Kopp, U. Ramsauer, Verwaltungsverfahrensgesetz: Kommentar, $\$ 39$, ${ }^{\circ}{ }^{\circ} 1$ sq.

61. BVerwGE (22 février 1985), 71, 63 (72).

62. BVerwG (22 janvier 1993), NJW, 1993, 1667 (1669).

63. BVerwGE (17 juillet 1980), 60, 297 (298). 
comme élément déterminant ${ }^{64}$. Considéré comme déterminant, le but oriente ainsi la motivation de la décision que l'autorité administrative doit réaliser ${ }^{65}$.

Si la motivation est susceptible de degrés, des exceptions subsistent pour lesquelles elle n'est pas exigée.

\section{Les exceptions à l'exigence de motivation}

Ces exceptions sont prévues au $\$ 39$ II VwVfG, lequel prévoit cinq cas. Les cas 4 et 5 peuvent déjà être évacués. Le cas 4 prévoit qu'une prescription peut expressément exclure toute motivation ${ }^{66}$ lorsqu'elle pourrait amener l'autorité administrative à divulguer des éléments susceptibles de porter atteinte à la sécurité de l'État fédéral ou d'un État fédéré ${ }^{67}$. Le cas 5 prévoit que la motivation n'est pas requise dès lors que l'acte constitue un acte réglementaire à caractère général et impersonnel ${ }^{68}$. Ces deux cas obligent à souligner que, non seulement la motivation n'est pas un principe absolu, mais, par ailleurs, qu'elle peut ne pas être exigée; au surplus, si le cadre général de la motivation est aménagé au sein du $₫ 39$ VwVfG, des dispositions aménageant un cadre dérogatoire de motivation subsistent pour des domaines particuliers ${ }^{69}$.

Les exceptions à l'exigence de motivation ne doivent pas être uniquement perçues comme une exclusion de celle-ci, mais comme autorisant aussi une motivation partielle, voire atténuée. Contrairement aux développements antérieurs où les cas d'atténuation concernaient la nature du pouvoir ou de la compétence reconnus à l'autorité administrative ${ }^{70}$, il s'agit, présentement, d'atténuations qui interviennent dans le cadre général même de la motivation et demeurent sans lien avec la nature du pouvoir de l'autorité administrative.

L'admission de ces exceptions pose évidemment des difficultés au regard du principe de l'État de droit, du principe de démocratie et de la sécurité juridique, à la concrétisation desquels la motivation est censée participer ${ }^{71}$.

En premier lieu, les caractéristiques des exceptions à l'exigence de motivation doivent être exposées. Tout d'abord, ces exceptions ne sont admises, d'une part, en présence de droits fondamentaux, que si le législateur a prévu, par ailleurs, un autre mécanisme pour leur protection et, d'autre part, que dans le cas où l'intérêt général emporte un poids tel que cela justifie l'exception ${ }^{72}$. Ensuite, les exceptions aménagées sont d'interprétation stricte et ne trouvent pas à s'appliquer en cas de «décision rendue sur contredit» (Widerspruchbescheid) ${ }^{73}$. Le recours sur contredit (Widerspruch) constitue un recours administratif préalable obligatoire au recours en annulation (Anfechtungsklage). Les exceptions à la motivation ne s'appliquent pas à la décision qui résulte de ce recours. Et pour cause, celui-ci constitue une phase préalable d'échange et de discussion entre l'administré et l'administration avant la saisine du juge. Cette phase et l'échange qui la caractérise sont censés permettre à l'autorité administrative d'expliquer le sens même de la décision qu'elle a adoptée, afin que l'intéressé puisse évaluer l'opportunité de saisir ou non le juge administratif. Appliquer les exceptions de motivation à la décision rendue sur contredit aurait traduit une incohérence certaine. La Cour constitutionnelle fédérale ${ }^{74}$ est venue ôter tout doute sur ce point, en précisant qu'en cas de contredit, l'autorité administrative est tenue d'énoncer les motifs déterminants ayant conduit à l'adoption de la décision. Enfin, les exceptions ne sont pas admises dans les procédures d'urgence. L'exigence de motivation persiste, mais s'articule en une motivation succincte qui doit rester cohérente ${ }^{75}$.

En second lieu, il convient de cerner les cas dans lesquels l'exception de motivation s'applique.

Tout d'abord, l'exception de motivation avait été admise par la cour administrative d'appel de Lunebourg ${ }^{76}$

64. BVerwGE (22 février 1985), 71, 63 (72).

65. BVerwG (22 janvier 1993), NJW, 1993, 1667 (1669).

66. Le $\$ 39$ II VwVfG dispose ainsi qu’ «Une motivation n’est pas nécessaire [...] 4. si cela découle d’une prescription juridique» («Einer Begründung bedarf es nicht, $[\ldots]$ 4. wenn sich dies aus einer Rechtsvorschrift ergibt»).

67. BVerwGE (29 octobre 1982), 66, 233; BVerwGE (19 août 1986), 75, 1; T. Müller-Ibold, Die Begründungspflicht..., p. 186.

68. \$39 II VwVfG: «Einer Begründung bedarfes nicht, [...] 5. wenn eine Allgemeinverfügung öffentlich bekannt gegeben wird». F.-O. Kopp, U. Ramsauer, Verwaltungsverfahrensgesetz: Kommentar, $\$ 39, \mathrm{n}^{\circ}$ 54-55.

69. Notamment en matière d'urbanisme: $\$ 74$ I phr. 2 VwVfG; en matière de demande d'asile: $\$ 31$ I 2 de la loi relative à l'asile $($ Asylgesetz - AsylG); en matière de pollution environnementale: $\$ 11 \mathrm{I}$ de la loi relative à la pollution environnementale (Umweltschadensgesetz - UschadG)...

70. Voir les développements du II.B.

71. P. Stelkens, H. J. Bonk, M. Sachs et al., Verwaltungsverfahrensgesetz: Kommentar, $\$ 39, \mathrm{n}^{\circ} 115 ;$ K. Obermayer, M. Funke-Kaiser, Kommentar zum $V w V f G, 5^{\mathrm{e}}$ éd., Munich, Luchterhand, 2018, $\$ 39, \mathrm{n}^{\circ} 43$; J. Ziekow, Verwaltungsverfahrensgesetz: Kommentar, $\$ 39, \mathrm{n}^{\circ} 7$.

72. F.-O. Kopp, U. Ramsauer, Verwaltungsverfahrensgesetz: Kommentar, «Einführung I», $\mathrm{n}^{\text {os }} 1-9$, en particulier $n^{\circ} 5$.

73. Bien qu'il s'agisse d’un recours obligatoire, le $\$ 68$ I VwGO en restreint la portée, principalement en cas de: prescription spéciale; lorsqu’une autorité administrative fédérale ou fédérée est l'auteur de l'acte administratif querellé; lorsque l'acte administratif a été adopté en matière de planification urbanistique ou lorsque l'intéressé ayant intenté ce recours préalable voit finalement ses droits subir une atteinte en raison du recours préalable ainsi intenté. Voir J.-M. Auby, M. Fromont, Les recours contre les actes administratifs dans les pays de la Communauté économique européenne: Allemagne, Belgique, France, Italie, Luxembourg, Pays-Bas, Paris, Dalloz, 1971, p. 42-52; R. Wakote, Le contrôle du pouvoir discrétionnaire..., p. 222 sq.

74. BVerfGE (16 janvier 1957), 6, 45 (49 sq.) (Staat als Beschwerdeführer); J. Ziekow, Verwaltungsverfahrensgesetz: Kommentar, $\$ 39, \mathrm{n}^{\mathrm{o}} 7$. Cette solution était bienvenue car elle avait aussi le mérite d'éviter les recours superflus des justiciables contre les décisions rendues sur contredit, retardant d'autant l'adoption de l'acte administratif définitif.

75. T. Müller-Ibold, Die Begründungspflicht..., p. 220.

76. OVG Lunebourg (Basse-Saxe - Schleswig-Holstein), 15 février 1989, $N V w Z, 1989$, p. 1181 et $D V B l, 1989$, p. 1106. 
au motif qu'elle provenait d'une demande de l'intéressé. Mais cette jurisprudence a été fortement critiquée par la doctrine ${ }^{77}$. En effet, comme cela a été évoqué, la motivation n'est pas aménagée en elle-même dans l'intérêt du destinataire de l'acte, mais dans une perspective de concrétisation des principes de l'État de droit, de sécurité juridique et de démocratie. La Cour administrative constitutionnelle a d'ailleurs rejeté cette solution: la motivation demeure une exigence, même lorsque le requérant affirme y renoncer. Elle admet seulement que la motivation puisse être sommaire, si et seulement si l'autorité administrative a déjà eu à se prononcer à l'occasion d'une décision antérieure dont les faits seraient similaires ${ }^{78}$. Par ailleurs, cette motivation sommaire ne fait pas obstacle à ce que l'intéressé en sollicite l'approfondissement dans le cadre du procès ${ }^{79}$. Aussi, une part de la doctrine en a déduit que l'intéressé peut renoncer à la motivation lorsque l'acte lui est favorable ${ }^{80}$. Mais là encore cette lecture ne semble pas conforme à l'esprit du $\$ 39$ VwVfG: non seulement la motivation n’est pas aménagée dans l'intérêt de l'intéressé, mais, par ailleurs, l'exigence de motivation s'applique sans prendre en considération le caractère favorable ou défavorable de l'acte.

Ensuite, l'exception de motivation est admise pour faciliter l'action administrative ou encore pour des «motifs d'économie administrative ${ }^{81}$. Toutefois cette justification s'inscrit en contradiction avec l'objectif de bonne administration poursuivi par la motivation aménagée au sein du $₫ 39$ VwVfG.

D’autre part, le $\$ 39$ VwVfG aménage dans le cas 1 l'exclusion de la motivation, lorsque l'acte administratif a été adopté sur demande ou déclaration de l'intéressé dès lors que, par ailleurs, la décision ne porte pas atteinte aux droits des tiers ${ }^{82}$. Cette approche est soutenue par une partie de la doctrine ${ }^{83}$. Les déclarations s'entendent au sens des $\$ 22$ et $65 \mathrm{VwVfG}$. Le $\$ 22 \mathrm{VwVfG}$ dispose que si l'autorité administrative décide, conformément à son pouvoir discrétionnaire, si et quand elle peut engager une procédure administrative, cette disposition ne s'applique toutefois pas lorsque d'une part l'autorité administrative, en application d'une prescription textuelle, doit agir d'office ou sur demande et, d'autre part, lorsque bien qu'autorisée à n'agir que sur demande, cette demande n’existe pas. Quant au $₫ 65 \mathrm{VwVfG}$, il aménage la prise en considération par l'autorité administrative des déclarations provenant de témoins ou d'experts ${ }^{84}$. Mais le refus de motivation en cas de déclaration tel que prôné par une partie de la doctrine et validé par la jurisprudence administrative ${ }^{85}$ semble critiquable, sur le plan constitutionnel ${ }^{86}$. Car cette situation n'exclut pas que l'absence de motivation puisse s'avérer, finalement, irrégulière si l'autorité administrative disposait, en réalité, d'un pouvoir discrétionnaire ou encore si les déclarations de l'intéressé ont été formulées sur un terrain de mécompréhension ou d'incompréhension de la situation ${ }^{87}$. Si la motivation peut être exclue, en cas de demande de l'intéressé, dès lors que cela ne porte pas atteinte aux droits des tiers, la question demeure, toutefois, de savoir si la motivation ne doit alors être opérée qu'à l'égard du tiers en question ou si elle doit aussi l'être à l'égard de l'intéressé dont les déclarations ou la demande ont provoqué l'acte adopté. La doctrine est encline, majoritairement, à penser que seul le tiers doit alors bénéficier d'une motivation, dès lors que ce sont les déclarations de l'intéressé qui sont à la base de la décision ${ }^{88}$. L'exclusion de la motivation n'est donc pas absolue en cas de décision adoptée sur demande ou sur déclaration; elle est juste limitée dans sa portée.

Enfin, le défaut de motivation d'une décision peut être «ignoré» (unbeachtet sein) lorsque cela n'a emporté aucune conséquence sur le contenu et le sens mêmes de la décision. Tel est le cas lorsqu'en cas de pouvoir discrétionnaire la prise en considération des droits fondamentaux aboutit à une "réduction à zéro du pouvoir discrétionnaire" (Ermessensreduktion auf Null), de sorte qu'avec ou sans la motivation, une seule décision s'imposait à l'autorité administrative; en l'occurrence celle qu'elle a adoptée sans motivation ${ }^{89}$. Il en va de même

77. F.-O. Kopp, U. Ramsauer, Verwaltungsverfahrensgesetz: Kommentar, «Einführung I», $\mathrm{n}^{\text {os }} 1-9$ et $\mathrm{n}^{\circ} 34$.

78. BVerwG, ordonnance du 19 août 1997, Buchholz 421.o (contrôle en matière d'examen), nº 342, p. 55; BVerwGE (14 octobre 1965), 22, 215 (217), BVerwGE (15 juin 1971), 38, 191 (194). Pour les critiques doctrinales, voir T. Müller-Ibold, Die Begründungspflicht..., p. 188 sq.; H.-J. Knack, H.-G. Henneke, Verwaltungsverfahrensgesetz: Kommentar, $\$ 39, \mathrm{n}^{\circ} 60$.

79. BVerwG (9 mai 1985), NVwZ, 1986, p. 375.

80. H.-J. Knack, H.-G. Henneke, Verwaltungsverfahrensgesetz: Kommentar, \$ 39, $\mathrm{n}^{\circ} 46$.

81. T. Müller-Ibold, Die Begründungspflicht..., p. 153; J. Lücke, Begründungszwang und Verfassung: zur Begründungspflicht der Gerichte, Behörden und Parlamente, Tübingen, Mohr Siebeck, 1987, p. 102.

82. Il peut s'agir d'intérêt juridiquement protégé, mais l'atteinte aux droits des tiers ne peut être de nature purement économique: BVerwGE (27 mars 1963), 16, 26 (27); F.-O. Kopp, U. Ramsauer, Verwaltungsverfahrensgesetz: Kommentar, $\$ 28, \mathrm{n}^{\circ} 24$ et $\$ 39, \mathrm{n}^{\circ} 38$.

83. H.-J. Knack, H.-G. Henneke, Verwaltungsverfahrensgesetz: Kommentar, $\$ 39, \mathrm{n}^{\circ} 36$.

84. Au sens des $\$ 22$ et $65 \mathrm{VwVfG}$, constitue une demande ou déclarations, les énonciations de l'intéressé permettant à l'autorité administrative de tirer des conclusions opportunes ou encore de cerner ses attentes. La demande ou déclaration doit donc être précise et circonstanciée; elle ne peut être accompagnée de réserves; elle ne peut pas reposer sur l'exercice du pouvoir discrétionnaire de l'autorité administrative; voir H.-J. Knack, H.-G. Henneke, Verwaltungsverfahrensgesetz: Kommentar, $\$ 39, \mathrm{n}^{\circ} 36$.

85. BVerwGE (29 juin 1967), 27, 253; BVerwGE (27 mars 1963), 16, 26 (27).

86. K. Obermayer, Grundzüge des Verwaltungsrechts..., p. 109; pour une position divergente, voir R. Dechsling, «Rechtsschutz und Begründungspflicht», DÖV, 1985, p. 718 .

87. R. Dechsling, «Rechtsschutz...», p. 714

88. J. Ziekow, Verwaltungsverfahrensgesetz: Kommentar, $\S_{39}, \mathrm{n}^{\circ} 9$; M. Fehling, B. Kastner, R. Störmer, Verwaltungsrecht, VwVfG, VwGO, Nebengesetze, Handkommentar, $4^{\mathrm{e} e ́ d ., ~ B a d e n-B a d e n, ~ N o m o s, ~ 2016, ~} \$ 39, \mathrm{n}^{\circ} 38$; P. Stelkens, H. J. Bonk, M. Sachs et al., Verwaltungsverfahrensgesetz: Kommentar, $\$ 39, n^{\circ} 83$. Pour une doctrine divergente, voir T. Müller-Ibold, Die Begründungspflicht..., p. 176.

89. F.-O. Kopp, U. Ramsauer, Verwaltungsverfahrensgesetz: Kommentar, $\$ 39, \mathrm{n}^{\circ} 59$. 
en cas de «pouvoir discrétionnaire orienté» (intendiertes Ermessen $)^{90}$.

En troisième lieu, le $\$ 39$ II VwVfG aménage en cas 2 l'exclusion de la motivation lorsque

[...] le destinataire de l'acte administratif ou celui concerné par l'acte administratif a déjà connaissance des éléments de fait et de droit à la base de la conception de l'autorité administrative ou [s'il] peut appréhender, sans difficulté, ces éléments en l'absence de motivation ${ }^{91}$.

Cette disposition aménagerait un cas d'exclusion de la motivation lié, pour reprendre une terminologie française, à la «connaissance acquise». Toutefois, cette connaissance acquise serait applicable tant à l'égard de l'intéressé ou du destinataire de l'acte qu'à l'égard des tiers. Or, étonnamment, la doctrine considère que l'autorité administrative doit être particulièrement attentive à l'égard des tiers, de sorte que ceux-ci puissent, intellectuellement, saisir le sens et la portée de la motivation ${ }^{92}$. Cette conception peut surprendre, car rien ne justifie qu'une telle exigence ne prévale pas aussi à l'égard de l'intéressé ou du destinataire de l'acte. Mais il est vrai que cette précaution est seulement préconisée lorsque la motivation repose sur une demande ou une déclaration de l'administré, considéré alors comme intéressé à l'adoption de l'acte.

Finalement, les cas 1 et 2 semblent peut-être bien plus proches qu'il n'y paraît: selon le cas 1, la motivation n'est pas nécessaire si l'acte a été adopté sur demande ou déclaration de l'intéressé, sous réserve des droits des tiers, et, selon le cas 2, la motivation est exclue si l'intéressé connaissait déjà les éléments de fait et de droit à la base de la décision, mais la doctrine et la jurisprudence rajoutent une attention particulière que l'autorité administrative doit manifester à l'égard du tiers. Si cette attention particulière manifestée à l'égard du tiers devait être admise, elle ne se justifierait qu'au regard de l'atteinte éventuelle à ses droits. De sorte, en définitive, qu'il conviendrait de retenir, pour les cas 1 et 2, que la motivation est exclue, lorsque l'acte a été adopté sur demande ou déclaration de l'intéressé ou lorsque l'intéressé peut se voir appliquer la théorie de la connaissance acquise par rapport aux éléments de fait et de droit à la base de la décision.

En dernier lieu, le $\$ 39$ II VwVfG aménage, en cas 3 , l'exclusion de la motivation, lorsque

[...] l'autorité administrative édicte des actes administratifs identiques en grand nombre ou les édicte par des moyens automatiques et que les circonstances de l'espèce ne nécessitent pas de motivation [de tels actes] ${ }^{93}$.
Au regard du $\$ 39 \mathrm{II}_{3}$, lorsque l’autorité administrative adopte une «multitude d'actes» par voie électronique, la motivation est soumise aux circonstances de l'espèce. Il $\mathrm{y}$ a lieu de ne pas exclure de prime abord de tels actes. Toutefois, la «multitude d'actes» ne suppose pas que les actes en cause aient été adressés simultanément à leurs destinataires. Ils peuvent avoir été envoyés de manière continue sur une longue période, voire sur une période indéterminée par avance. Enfin, un nombre minimum d'envois n'est pas requis: la multitude s'établit dans la durée ${ }^{94}$. Si, initialement, de tels actes étaient dispensés de motivation, le développement du recours à l'outil informatique et la crainte de voir l'autorité administrative recourir plus fréquemment à ce mode communication pour faire connaître ses décisions ont renforcé les exigences en la matière ${ }^{95}$. Ces actes ne sont donc plus totalement exclus de l'exigence de motivation. S'ils peuvent l'être, le $\$ 39$ II 3 précise que la dispense de motivation dépend des «circonstances de l'espèce» (voir II.B).

Au regard de ces développements, il y a lieu de retenir que le droit allemand ne proclame pas un principe général de motivation des actes administratifs. Celle-ci demeure non seulement susceptible de variations, de degrés, mais fait aussi l'objet d'exceptions, la jurisprudence de la Cour administrative fédérale s'efforçant d'intégrer les circonstances de l'espèce.

Dès lors, il convient d'aborder la portée de la motivation ainsi mise en œuvre.

\section{La portée de l'exigence de motivation}

Pour cerner la portée de l'exigence de motivation, en droit allemand, il convient de souligner que celui-ci distingue entre l'irrégularité (Unregelmäßigkeit) et la nullité (Nichtigkeit) de l'acte administratif en cause (A). Cette distinction autorise la "guérison» (Heilung) ou le «rétablissement» (Nachholung) de l'acte administratif dépourvu de motivation ou doté d'une motivation irrégulière (B).

\section{A. La distinction entre l'irrégularité et la nullité de l'acte administratif dépourvu de motivation}

La violation de l'exigence de motivation conduit à considérer l'acte irrégulier (unregelmäßig); cependant, celui-ci n'est pas nul (nichtig). L'irrégularité s'entend alors, en droit allemand, comme un vice formel lié à un défaut de

90. OVG Greifswald (Mecklenburg-Vorpommern), 19 janvier 2016, $N V w Z-R R, 2016$, p. 729.

91. \$39 II VwVfG: «Einer Begründung bedarf es nicht, [...] 2. soweit demjenigen, für den der Verwaltungsakt bestimmt ist oder der von ihm betroffen wird, die Auffassung der Behörde über die Sach- und Rechtslage bereits bekannt oder auch ohne Begründung für ihn ohne weiteres erkennbar ist ».

92. T. Müller-Ibold, Die Begründungspflicht..., p. 178; F.-O. Kopp, U. Ramsauer, Verwaltungsverfahrensgesetz: Kommentar, $\$ 39$, no 43.

93. \$39 II VwVfG: «Einer Begründung bedarf es nicht, [...] 3. wenn die Behörde gleichartige Verwaltungsakte in größerer Zahl oder Verwaltungsakte mit Hilfe automatischer Einrichtungen erlässt und die Begründung nach den Umständen des Einzelfalls nicht geboten ist ».

94. F.-O. Kopp, U. Ramsauer, Verwaltungsverfahrensgesetz: Kommentar, $\$ 39, \mathrm{n}^{\circ} 46$.

95. En 2002, un $\$ 3$ a a été inséré au Code de procédure administrative (VwVfG), procédant ainsi à un premier aménagement de l'administration numérique. Plus récemment, la loi relative à l'administrative numérique a été adoptée, en 2013, pour fixer le cadre du recours à l'informatique dans les échanges des autorités administratives avec les administrés; loi du 25 juillet 2013, BGBl. I, 31 juillet 2013 , p. 2749. 
motivation ou à une motivation insuffisante qui n'affectent pas la régularité de l'acte sur le fond. L'acte administratif est présumé «valide» (rechtsgültig) sur le fond, c'est-àdire eu égard à l'application des normes juridiques sur le fondement desquelles il a été adopté. Le contrôle du vice lié à la motivation ne confine pas à un contrôle des éventuels vices liés à l'erreur de droit.

Il en résulte que la motivation est irrégulière, principalement lorsque, bien que l'autorité administrative y a procédé, elle n'a pas, en réalité, indiqué les éléments de fait et de droit sur lesquels elle s'est véritablement fondée pour adopter l'acte critiqué ${ }^{6}$. Ce faisant, le défaut de motivation ou une motivation irrégulière ne fait pas courir les délais de recours juridictionnel.

Toutefois, l'irrégularité et la nullité de l'acte peuvent simultanément coexister, si et seulement si l'irrégularité relative à la motivation, couplée avec des éléments autres que l'erreur de droit résultant des normes applicables, traduit le caractère arbitraire de l'acte administratif. Une autre particularité subsiste, en droit allemand, en cas de pouvoir discrétionnaire reconnu à l'autorité administrative: dans ce cas, une motivation irrégulière aboutit non seulement à un exercice irrégulier du pouvoir discrétionnaire ainsi reconnu, mais aussi à l'irrégularité matérielle de la décision administrative ${ }^{97}$. Il y a alors irrégularité de l'acte et sur le plan formel et sur le plan matériel, sans que pour autant cela n'emporte sa nullité!

En effet, la nullité de l'acte ne peut être retenue, puisque le vice lié à la motivation qui l'entache peut faire l'objet d'une "guérison» (Heilung), permettant ainsi le «rétablissement» (Nachholung) de l'acte.

\section{B. Le possible "rétablissement" de la motivation ou la "guérison" de l'acte administratif}

Le rétablissement de l'acte administratif irrégulier est aménagé au $\$ 45 \mathrm{I} 2 \mathrm{VwVfG}$ en ces termes:

La violation des exigences procédurales ou formelle qui n’entraîne pas la nullité de l'acte en vertu du $\$ 44$ VwVfG est sans objet si $[\ldots]$ 2. les motifs peuvent être présentés ultérieurement $[\ldots]^{98}$.

En premier lieu, dès lors que l'atteinte portée à l'exigence de motivation s'analyse en une irrégularité et non en une nullité, l'exigence peut être satisfaite, ultérieurement, en énonçant la motivation de l'acte administratif... et ce après qu'il a été adopté.
Toutefois ne peuvent être rétablis que les éléments de fait et de droit qui auraient permis de satisfaire, effectivement, à la motivation de la décision et donc son adoption. En ce sens la motivation ultérieure ne doit pas emporter modification du contenu et du sens matériels de la décision.

En deuxième lieu, le destinataire de l'acte ne peut pas exiger le rétablissement de la motivation, ni solliciter que la motivation déjà énoncée soit davantage développée ${ }^{99}$. Cela est toutefois critiqué par une partie de la doctrine qui estime que cette exigence doit être admise ${ }^{100}$. Mais le refus s'appuie sur le fait que le principe de motivation sert avant tout la concrétisation de l'État de droit et non les intérêts du destinataire de l'acte. Une exception est cependant admise, lorsque la motivation est intervenue dans les conditions de l'urgence (voir II.C). En réalité, seule l'autorité administrative peut procéder, de sa seule initiative, à un rétablissement de la motivation. Il en résulte qu'elle devra assumer les frais de procédure, si ce rétablissement, intervenant soit dans le cadre de la procédure sur contredit soit dans la phase juridictionnelle, aboutit, finalement à l'extinction de tout contentieux ${ }^{101}$.

En dernier lieu, il convient de distinguer le « rétablissement de la motivation» (Nachholung der Begrüdung) de «l'invocation a posteriori de motifs » (Nachschieben von Gründen). Ce second cas n'est admis qu'en présence d'un pouvoir discrétionnaire reconnu à l'autorité administrative. Dans ce cas, l'administration a procédé à un usage déficitaire de son pouvoir discrétionnaire, c'està-dire qu'elle n'a pas pleinement apprécié les éléments de fait et de droit en intégrant le fait qu'elle disposait d'un tel pouvoir. Cette situation se rapporterait, selon la terminologie du droit français, à l'incompétence négative de l'autorité administrative. Convaincue de ne pas détenir un pouvoir discrétionnaire, elle a adopté sa décision dans un sens donné et l'a motivée en conséquence. Mais si, ultérieurement, elle réalise avoir disposé, initialement, d'un tel pouvoir, lui permettant d'adopter une autre décision, elle est admise, à invoquer a posteriori des motifs qu'elle avait écartés ou méconnus antérieurement ${ }^{102}$.

En droit allemand, l'exigence de motivation a été affirmée bien plus précocement qu'en droit français, puisque la loi de 1976 portant codification de la procédure administrative a rassemblé, en les synthétisant, les solutions jurisprudentielles antérieures. Toutefois la motivation ne constitue pas, comme en droit français, un principe général. Elle subit des atténuations et les circonstances en permettent, parfois, l'accentuation. Par ailleurs, des

96. F.-O. Kopp, U. Ramsauer, Verwaltungsverfahrensgesetz: Kommentar, $\$ 39, \mathrm{n}^{\circ} 56$.

97. H.-J. Knack, H.-G. Henneke, Verwaltungsverfahrensgesetz: Kommentar, \$ 39, nº 61; VGH Mannheim, 27 février 2014, DVBl, 2014, p. 604.

98. $\$ 45$ I 2 VwVfG: «Eine Verletzung von Verfahrens- oder Formvorschriften, die nicht den Verwaltungsakt nach $\$ 44$ nichtig macht, ist unbeachtlich, wenn $[\ldots]$ 2. die erforderliche Begründung nachträglich gegeben wird [...]».

99. BVerwGE (9 novembre 1984), 70, 247.

100. H.-J. Knack, H.-G. Henneke, Verwaltungsverfahrensgesetz: Kommentar, $\$ 39, \mathrm{n}^{\circ} 64$.

101. F.-O. Kopp, U. Ramsauer, Verwaltungsverfahrensgesetz: Kommentar, $\$ 39, \mathrm{n}^{\circ} 58$.

102. BVerwG (5 septembre 2006), NVwZ, 2007, p. 470. 
domaines de l'action administrative font l'objet d'un aménagement particulier.

Mais il convient de relever qu'en droit allemand la motivation n'a pas été instaurée dans la perspective d'une plus grande protection des intérêts des citoyens. Elle vise d'abord à concrétiser les principes d'État de droit, de démocratie et de sécurité juridique ainsi qu’à asseoir une «bonne administration». Cette conception explique aussi de manière sous-jacente l'impossibilité pour l'intéressé de solliciter une telle motivation, lorsque celle-ci s'est avérée irrégulière ou déficitaire. Elle justifie aussi, parallèlement, la possibilité reconnue à la seule autorité administrative d'en entreprendre le «rétablissement» et, par voie de conséquence, la "guérison» de l'acte. En définitive, il ne semble pas que le cadre de la motivation aménagé, en droit allemand, soit plus protecteur que celui préexistant en droit français : l'acte n'encourant aucune annulation, en dépit d'un vice affectant la motivation et pouvant toujours être "guéri», même dans la phase juridictionnelle.

\title{
Liste des sigles utilisés dans les références en notes de bas de page
}

\author{
BGBl. Bundesgesetzblatt \\ (journal officiel fédéral, Allemagne) \\ $\mathrm{BGBl}$. I renvoie à la première partie consacrée au droit interne, \\ tandis que BGBl. II fait référence à la seconde partie consacrée au droit international. \\ BVerfGE Entscheidungen des Bundesverfassungsgerichts \\ (recueil des décisions de la Cour constitutionnelle fédérale)* \\ BVerwG Bundesverwaltungsgericht \\ (Cour administrative fédérale) \\ BVerwGE Entscheidungen des Bundesverwaltungsgerichts \\ (recueil des décisions de la Cour administrative fédérale)* \\ DÖV Die Öffentliche Verwaltung (revue) \\ DVBl Deutsches Verwaltungsblatt (revue) \\ NJW Neue Juristische Wochenschrift (revue) \\ NVwZ Neue Zeitschrift für Verwaltungsrecht (revue) \\ NVwZ-RR Neue Zeitschrift für Verwaltungsrecht-Rechtsprechungs-Report (revue) \\ OVG Oberverwaltungsgericht \\ (Tribunal administratif supérieur) \\ RÜ2 Rechtsprechungsübersicht 2 (revue) \\ VGH Verwaltungsgerichtshof \\ (cour administrative régionale) \\ VwGO Verwaltungsgerichtsordnung \\ (loi relative à la juridiction administrative, 21 janvier 1960) \\ VwVfG Verwaltungsverfahrensgesetz \\ (loi relative à la procédure administrative non contentieuse, 25 mai 1976)
}

\footnotetext{
* Pour les arrêts et décisions cités, les références indiquées renvoient d'abord au numéro du volume, puis à la page où figure l'arrêt ou la décision et, enfin, entre parenthèses, à la page où figure le passage relevé ou cité.
} 